\title{
Non-Intrusive Load Monitoring Assessment: Literature Review and Laboratory Protocol
}

RS Butner

DJ Reid

M Hoffman

G Sullivan

J Blanchard

July 2013

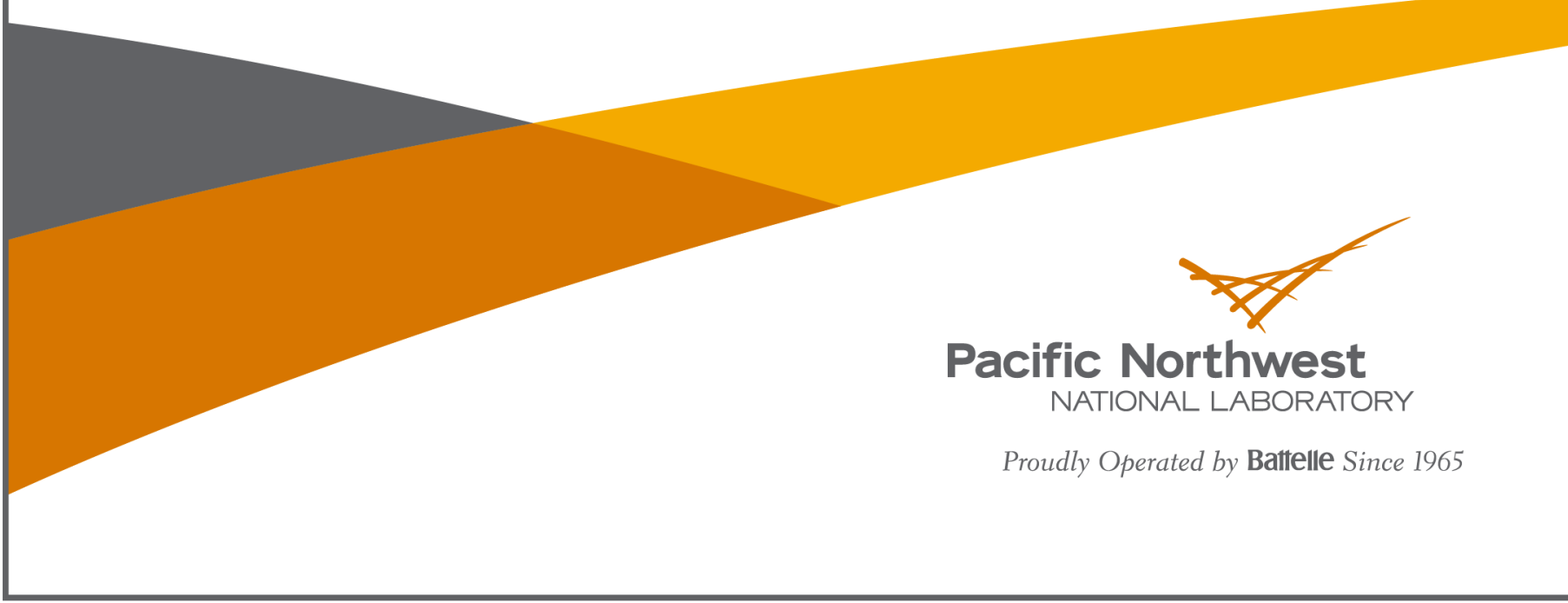




\title{
DISCLAIMER
}

This report was prepared as an account of work sponsored by an agency of the United States Government. Neither the United States Government nor any agency thereof, nor Battelle Memorial Institute, nor any of their employees, makes any warranty, express or implied, or assumes any legal liability or responsibility for the accuracy, completeness, or usefulness of any information, apparatus, product, or process disclosed, or represents that its use would not infringe privately owned rights. Reference herein to any specific commercial product, process, or service by trade name, trademark, manufacturer, or otherwise does not necessarily constitute or imply its endorsement, recommendation, or favoring by the United States Government or any agency thereof, or Battelle Memorial Institute. The views and opinions of authors expressed herein do not necessarily state or reflect those of the United States Government or any agency thereof.

\author{
PACIFIC NORTHWEST NATIONAL LABORATORY \\ operated by \\ BATTELLE \\ for the \\ UNITED STATES DEPARTMENT OF ENERGY \\ under Contract DE-AC05-76RL01830
}

Printed in the United States of America

Available to DOE and DOE contractors from the

Office of Scientific and Technical Information,

P.O. Box 62, Oak Ridge, TN 37831-0062;

ph: (865) 576-8401

fax: $(865) 576-5728$

email: reports@adonis.osti.gov

Available to the public from the National Technical Information Service

5301 Shawnee Rd., Alexandria, VA 22312

ph: (800) 553-NTIS (6847)

email: orders@ntis.gov <http://www.ntis.gov/about/form.aspx>

Online ordering: http://www.ntis.gov 


\title{
Non-Intrusive Load Monitoring Assessment: Literature Review and Laboratory Protocol
}

\author{
RS Butner \\ DJ Reid \\ M Hoffman \\ G Sullivan \\ J Blanchard
}

July 2013

Prepared for

the U.S. Department of Energy

under Contract DE-AC05-76RL01830

Pacific Northwest National Laboratory

Richland, Washington 99352 



\section{Executive Summary}

Unlike sub-metering, which requires that individual loads are equipped with their own meters, nonintrusive load monitors (NILM) use algorithms to discern the individual loads from a single metering point. Although a number of these technologies are now commercially available to utilities and homeowners, with several more to become available in the coming years, it is not yet understood how accurately these technologies can disaggregate a home's electrical load. Pacific Northwest National Laboratory (PNNL) and its partners at the Northwest Energy Efficiency Alliance (NEEA) have identified and acquired six of these technologies and will be testing them in the PNNL Lab Homes as well as the owner-occupied Residential Test Bed operated by NEEA. As a result of this testing, PNNL and NEEA will evaluate the selected NILMs against a repeatable test protocol so that their accuracy can be compared across NILM technologies. In the field deployment in the NEEA Test Bed Homes, PNNL and NEEA will evaluate the real-world accuracy of the NILMs responding to dynamic loads.

To evaluate the accuracy of NILM technologies regardless of their intended application, a standardized test protocol is required that applies representative loads and establishes consistent metrics against which the NILM can be evaluated and compared. To establish this standardized test protocol, a literature review was conducted to identify any test protocols or standardized testing approaches currently in use. The literature review indicated that no consistent conventions were currently in place for measuring the accuracy of these technologies. Consequently, PNNL developed a testing protocol and metrics to provide the basis for quantifying and analyzing the accuracy of commercially available NILM technologies. This report discusses the results of the literature review and the proposed test protocol and metrics in more detail. 



\title{
Acronyms and Abbreviations
}

\author{
CT current transformer \\ NILM nonintrusive load monitor \\ NEEA Northwest Energy Efficiency Alliance \\ PNNL Pacific Northwest National Laboratory \\ TED The Energy Detective
}





\section{Contents}

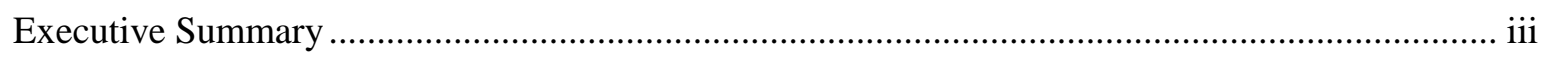

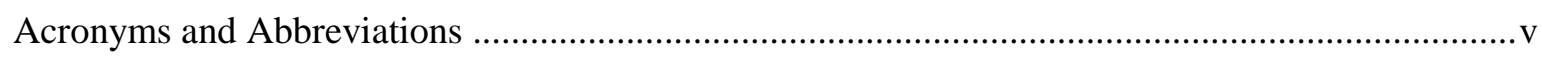

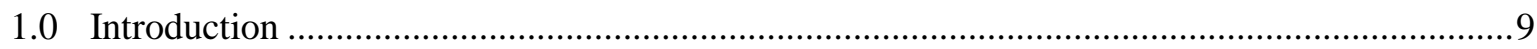

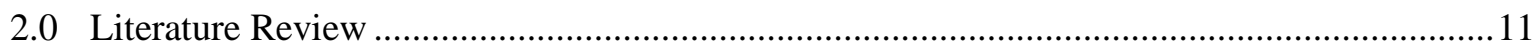

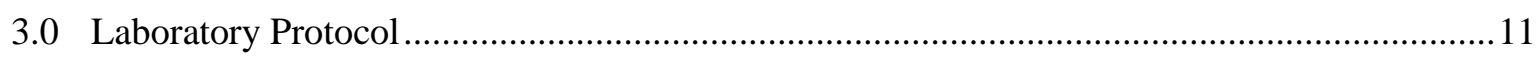

4.0 Appendix A. Literature Review: Relevant NILM Reports, Papers, and Presentations ........... 14

5.0 Appendix B. NILM Protocol Mechanics: Monitoring and Disaggregation Metrics ............... 18

6.0 Appendix C. Controllable Electrical Panel and Reference/Baseline Metering Equipment .....25

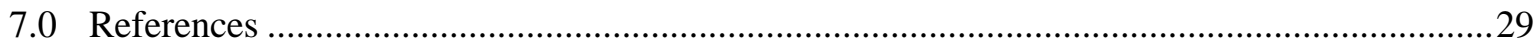




\section{Tables}

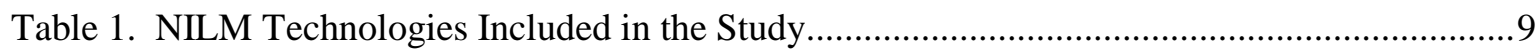

Table 2. NILM Testing Protocol: Monitoring and Disaggregation Metrics ................................... 12 


\subsection{Introduction}

This literature review and laboratory analysis satisfies deliverable number PNNL 13R1.06.1.2F within Project 6.1, NILM Assessment. Initially the PNNL team identified a number of candidate nonintrusive load monitoring (NILM) technologies available or under development for use in residential homes, with 16 in total, for inclusion in the study. From these 16 initial candidates, only 6 were ultimately selected for testing due to both price and availability.

While all these technologies are advertised as accomplishing the same end goal of disaggregating a home's total electrical load into its major components, they take different technical approaches for doing so. To facilitate the analysis, each of these technologies was sorted into one of three technology categories based on the methods they employ to disaggregate electrical loads: current transformer (CT) based devices (installed in the breaker panel), meter devices installed at the utility meter on the exterior of the home, and software-only-based solutions which rely on third-party hardware to collect and transmit the data to vendor servers where the data are accessed, processed, and presented as disaggregated energy use.

1) Current Transformer (CT) Based Devices: This category of device relies on field-installed CTs along with voltage taps to directly measure whole-house energy use. Load disaggregation takes place after data are uploaded to the vendor's servers where analytics are applied.

2) Utility-Meter-Based Devices: This category of device uses single-point metering installed at the utility meter, typically a collar or meter base to which the utility meter is installed. A major benefit of this technology is that it does not require installation in the customer's electrical panel. As with the CT-based devices, load disaggregation takes place after data are uploaded to the vendor's servers.

3) Software-Only Solutions: Software-only-based systems rely on third-party hardware to collect and transmit the energy-use data to a vendor's server where the load is disaggregated using the software algorithms.

The six technologies included in the final study featured two products of each type and are shown in Table 1 below.

Table 1. NILM Technologies Included in the Study

\begin{tabular}{|l|l|l|}
\hline Manufacturer & \multicolumn{1}{l|}{ Product Name } & \multicolumn{1}{l|}{ Technology Category } \\
\hline Belkin & Unnamed & Utility Meter Device \\
\hline Bidgley & Bidgley & Software-Only \\
\hline Energy Aware & PowerBlaster & CT-Based Device \\
\hline Energy, Inc. & TED 5000 & CT-Based Device \\
\hline Enetics & SPEED & Utility Meter Device \\
\hline PlottWatt & PlottWatt & Software-Only \\
\hline
\end{tabular}


More-detailed descriptions of the six technologies included in the PNNL and NEEA study are given below.

Belkin: Belkin is currently developing a NILM product that is designed to be installed at the external utility meter of a home, though the final commercial product may be installed in the breaker panel with CTs. This device then tracks and transmits load usage data to a central cloud server where the total load is disaggregated into its base components based on algorithms developed by Belkin. Users will eventually be able to access their data from a website-based dashboard that displays their electricity use by common appliances once the product is commercially released.

Bidgely: Bidgely offers a cloud-based software program that collects meter data from third-party hardware, such as The Energy Detective (TED). Bidgely does not manufacture hardware of their own, and uses analytical algorithms to disaggregate energy consumption. Once disaggregated, the software presents the user with recommended behavioral changes or appliance replacements to conserve electricity based on the disaggregated electricity consumption data. The Bidgely website reports that the software learns energy use behavior without any plug-level sensors, suggesting that the software has an automated learning capability and that there is no training period where the user must manually tell the unit which loads are operating. The algorithm also requests that users enter information about themselves to improve the accuracy of the disaggregation. The service is compatible with many other energy metering products (such as TED 5000 or Wattless), or with smart meters equipped with ZigBee® interfaces. For both the PNNL and NEEA test homes, data will be collected and sent to the Bidgley servers via panel-installed TED units.

The Energy Detective 5000 (TED 5000 ${ }^{\mathrm{TM}}$ ): A commercially available residential energy meter, the TED 5000 (versions are available for both single and three-phase) is installed in the breaker panel. The system is installed with CTs at the power mains into the panel and voltage taps at two unused breakers - one for each phase. Data are collected and delivered using power-line carrier technology through the home's existing electrical wiring to a gateway device connected to the home's internet service, which then uploads the data to a cloud-based server. The product combines both the metering hardware as well as a software application. In order to disaggregate the home's load, users must manually "teach" the program when certain appliances are operating based on event recognition. This product can reportedly disaggregate up to five appliances and produce energy conservation suggestions for the user based on energy use data.

Energy-Aware: The product, called the PowerBlaster ${ }^{\mathrm{TM}}$, combines metering hardware that is installed at the electrical panel (CTs and voltage taps similar to the TED), to measure energy use. Data are collected and transferred using power-line carrier technology to a gateway where they are uploaded to the company's servers. Once uploaded, the user can reportedly access and disaggregate the data via web-based analytical tools. The Energy-Aware product reportedly does not have a limit on the number of appliances it can disaggregate, and does not require the users to manually teach the algorithm which electrical loads are currently operating to improve the disaggregation.

Enetics: This device, called SPEED ${ }^{\mathrm{TM}}$ (Single Point End-use Energy Disaggregation), includes metering hardware and software packages for disaggregating loads and generating reports on individual appliance energy consumption. The system is designed to be installed at the home's external power meter coupled with an interior device that reads interior home temperature. Data collected by the meter is uploaded and processed via algorithms to determine the load distribution among appliances in the home. While the system is primarily intended for use by utilities or property management organizations, the company is reportedly interested in becoming involved in the home NILM market by producing a system that is intended to be directly used by homeowners. 
PlotWatt: PlottWatt has developed a software-only tool for disaggregating residential load data collected from a preexisting utility smart meter (such as WattVision) or panel-installed meter (such as TED $5000^{\mathrm{TM}}$ ). The product is currently available to the public for use with a limited number of smart meters and power meters, with more expected in the future. The software is free for residential users. Data collected by the meters is uploaded to the company's cloud-based server and then disaggregated.

\subsection{Literature Review}

Testing each of these technologies requires the development of testing protocols and metrics that measure the accuracy of load disaggregation, applicable to all three of the identified product groups. Prior to the development of these testing protocols, a literature review was conducted to assess what, if any, conventions presently exist for testing these technologies. This literature review was also intended to identify other laboratory or field evaluations of NILM devices and to survey the protocols and metrics used. A general finding of this literature review was that a consistent set of NILM evaluation metrics or protocols has not been previously developed or described in the literature. Interviews with technology manufacturers further reflected a lack of consensus with regard to NILM evaluation metrics, as many were themselves uncertain about what norms or best practices, if any, existed for testing NILM disaggregation results.

In all, over 50 technical papers, reports, presentations and research papers were identified and reviewed as part of this effort; Appendix A presents 35 of the most relevant documents reviewed. In addition, standard testing protocols for similar electrical metering equipment were examined. As an outcome of this review, three documents referencing protocols for testing and evaluating NILM technologies were identified to have relevance to this task (Ziefman et al. 2011, Zoha et al. 2012, and Liang et al., 2010).

Although not included in this literature review due to accessibility and expense challenges, the Electric Power Research Institute (EPRI) has reportedly conducted some laboratory assessments on the accuracy of four available residential NILM technologies. Their report, published in May, 2013, (copies may be purchased from EPRI for \$25,000) and includes a summary of available technologies, taxonomy of NILM technologies, and the results of EPRI's laboratory evaluations.

Given the absence of consensus within both the literature and from the manufacturers, a testing protocol was developed with input from both parties and following guidelines developed by Liang (Liang, et al. 2010) to create a series of metrics for product evaluation.

\subsection{Laboratory Protocol}

Due to the variation found in the available NILM technologies, testing protocols had to be developed to evaluate the NILM technologies in a consistent, equitable, and repeatable manner. Testing protocols were designed to be agnostic to the technical approach employed by each product, quantifying only the disaggregation accuracy while excluding any other considerations from the analysis (such as ease of use, price, etc.).

Specifically, this protocol was designed only for technical assessment purposes. The developed protocol will evaluate technologies along two primary dimensions. First, each technology will be tested 
with regard to the metering hardware that is installed in either the breaker panel or power meter itself (referred to here as "Monitoring Metrics"): the equipment's ability to correctly detect operational characteristics of a load, load magnitude, load duration, and coincidence of several loads simultaneously. In the case of the software solutions, the reliant metering hardware, while not part of the vendor product, will be assessed for performance.

The second metric is designed to evaluate the load disaggregation technology based on its disaggregation performance (denoted collectively as "Disaggregation Metrics"): specifically unique event detection, event disaggregation, overall event accuracy, event power use, energy share of total home load, and repeatability of performance. See Table 2 below for a list of the criteria under each metric.

Table 2. NILM Testing Protocol: Monitoring and Disaggregation Metrics

\begin{tabular}{|l|}
\hline \multicolumn{1}{|c|}{ Monitoring Metrics } \\
\hline Operational Characteristic \\
Load Magnitude \\
Load Type \\
Load Duration \\
Coincidence of Load \\
\hline
\end{tabular}

\begin{tabular}{|l|}
\hline \multicolumn{1}{|c|}{ Disaggregation Metrics } \\
\hline Event Detection \\
Event Disaggregation \\
Event Accuracy \\
Power Use \\
Energy Shares \\
Repeatability \\
\hline
\end{tabular}

Execution of these testing protocols will be conducted in in two stages: the first will occur in August and September, 2013, in the PNNL Lab Homes once all technologies have been acquired, installed, and configured. During this phase both homes will be used to evaluate the accuracy of the NILM regarding the aforementioned performance parameters using a series of simulated resistive (such as electric resistance heaters) and reactive (such as the motor in a washing machine) plug loads on a set schedule in accordance with the testing protocols over a two-week period. The second stage will be conducted in both the PNNL Lab Homes and the NEEA Residential Test Bed homes for approximately one year of data collection where the meters are allowed to run in the background during normal occupation of the homes and will be subject to the dynamic loads of typical household operation.

The initial two-week protocol testing period in the Lab Homes will be used to extensively test the technologies in a controlled setting to determine their sensitivity to and accuracy with different load types, magnitudes, durations, and frequencies, and under coincident loads, in a standardized manner. Meters installed in the NEEA Residential Test Bed Homes will not be subject to any specific test protocol or have any set duration, and will instead be used as the basis for assessing the accuracy of disaggregation under real-world conditions in owner-occupied homes. These initial tests will be used to make preliminary observations regarding the accuracy of each technology under controlled conditions. Appendix B presents the Lab Homes NILM test protocol in more detail.

The subsequent testing of each technology will be conducted by virtue of the meters remaining in place in both the PNNL Lab Homes and in the NEEA Residential Test Bed homes over the coming year and may serve as the basis of a broader analysis about the accuracy of their disaggregations over a prolonged period of time and over different occupancy patterns. The metering and sensor equipment used 
in the Lab Homes to measure the loads that are not directly associated with NILM technologies are covered in detail in Appendix C. 


\section{Appendix A. Literature Review: Relevant NILM Reports, Papers, and Presentations}

The reports, papers, and presentations below represent the relevant body of research reviewed as part of this project. While not all publications provided useful information to this specific effort, most contain pertinent information to the larger NILM topic.

Alahmad M, and H Hasna. 2011. "Non-Intrusive Electrical Load Monitoring and Profiling Methods for Applications in Energy Management Systems.” 2011 IEEE Long Island Systems, Applications and Technology Conference (LISAT). University of Nebraska, Lincoln, Nebraska. Accessed July 19, 2013 at http://digitalcommons.unl.edu/archengfacpub/49/.

Anderson KD, ME Berges, A Ocneanu, D Benitez, et al. 2012. "Event Detection for Non Intrusive Load Monitoring." IECON 2012 - $38^{\text {th }}$ Annual Conference on IEEE Industrial Electronics Society. Montreal Quebec, Canada. Accessed July 19, 2013 at http://ieeexplore.ieee.org/xpl/articleDetails.jsp?arnumber=6389367

Berges ME and E Goldman. 2010. "Enhancing Electricity Audits in Residential Buildings with Nonintrusive Load Monitoring." Journal of Industrial Ecology, Environmental Applications of Information \& Communication Technology 14(5): 844-858. Carnegie Mellon University, Pittsburgh, Pennsylvania. Accessed July 19, 2013 at http://onlinelibrary.wiley.com/doi/10.1111/j.1530$\underline{9290.2010 .00280 . x / a b s t r a c t}$

Berges M, E Goldman, H Matthews, H Scott, and L Soibelman. 2008. "Training Load Monitoring Algorithms on Highly Sub-Metered Home Electricity Consumption Data." Tsinghua Science and Technology 13(S1): 406-411. Tsinghua University Press, Beijing, China. Accessed July 19, 2013 at http://ieeexplore.ieee.org/xpl/articleDetails.jsp?reload=true\&arnumber=6073013\&contentType=Journals $+\% 26+$ Magazines

Carlson DR, M. Berges, and H. Matthews. 2012. "How Many Appliances Does it Take To...?" International Workshop on Non-Intrusive Load Monitoring. Department of Civil and Environmental Engineering, Carnegie Mellon University, Pittsburgh, Pennsylvania. Accessed July 19, 2013 at http://www.ices.cmu.edu/psii/nilm/abstracts/carlson_CMU_NILM2012_abstract.pdf

Cheng C, TJT Sung, GY Lin, and J.C Wen. 2011. "Non-intrusive Appliance Monitoring Now: Effective Data, Generative Modeling and LETE." In ENERGY 2011. The First International Conference on Smart Grids, Green Communications and IT Energy-aware Technologies. Accessed July 19, 2013 at http://www.thinkmind.org/index.php?view=article\&articleid=energy_2011_5_10_50074

Froehlich J, and E Larson, 2010. "Disaggregated End-Use Energy Sensing for the Smart Grid." Pervasive Computing/IEEE. Volume 10, Issue 1. University of Washington, Seattle, Washington, 2011. Accessed July 19, 2013 at http://ieeexplore.iee. $.0 r g / x p l s / a b s ~ a l l . j s p ?$ arnumber=5590234

Goldman E, and M Berges. "A real-world evaluation of NILM: tradeoffs between data collection and information effectiveness." PowerPoint presentation. Carnegie Mellon University, Pittsburgh,

Pennsylvania. Accessed July 19, 2013 at

http://www.ece.cmu.edu/ electricityconference/2009/2009\%20CMU\%20Smart\%20Grids\%20Conf\%20D 
isk/Presentations/Day\%202/Session\%204A/P1_E\%20Goldman\%20and\%20M\%20Berges_A\%20realworld\%20evaluation\%20of\%20NILM.pdf

Gomaton K, and C Holmes. 2012. "Evaluation of NILMs Technologies for Electric Load Disaggregation." PowerPoint presentation. First International Workshop on Non-Intrusive Load Monitoring. Carnegie Mellon University, Pittsburgh, Pennsylvania. Accessed July 19, 2013 at http://www.ices.cmu.edu/psii/nilm/slides/gomatom_EPRI_NILM2012_slides.pdf

Gray J. "Big Data Scale and Energy Insight." Verlitics, Portland, Oregon. Accessed July 19, 2013 at http://www.ices.cmu.edu/psii/nilm/abstracts/gray_Verlitics_NILM2012_abstract.pdf

Irwin D, A Wu S Barker, A Mishra, P Shenoy, and J Albrecht. "Exploiting Home Automation Protocols for Load Monitoring in Smart Buildings.” University of Massachusetts-Amherst, Amherst, Massachusetts; Amherst College, Amherst, Massachusetts; and Williams College, Williamstown, Massachusetts. Accessed July 19, 2013 at http://lass.cs.umass.edu/papers/pdf/buildsys11-insteon.pdf

Kolter JZ, and T Jaakkola. 2012. "NILM Beyond Event Detection.” PowerPoint presentation. 2012 NILM Workshop. MIT Computer Science and Artificial Intelligence Laboratory, Cambridge, Massachusetts. Accessed July 19, 2013 at http://www.ices.cmu.edu/psii/nilm/slides/kolter_MIT_NILM2012_slides.pdf

Kolter J, and MJ Johnson. "REDD: A Public Data Set for Energy Disaggregation Research.” Computer Science and Artificial Intelligence Laboratory, Laboratory for Information and Decision Systems. Massachusetts Institute of Technology, Cambridge, Massachusetts. Accessed July 19, 2013 at http://redd.csail.mit.edu/kolter-kddsust11.pdf

Kuhns H, M Roberts, and B Bastami. "Closure Rules for Non-Intrusive Appliance Load Monitoring." PowerPoint presentation. Reno, Nevada. Accessed July 19, 2013 at http://www.ices.cmu.edu/psii/nilm/slides/kuhns_LoadIQ_NILM2012_slides.pdf

Lai S, M Trayer, S Ramakrishna, and Y Li. "Database Establishment for Machine Learning in NILM." PowerPoint presentation. Samsung Telecommunications America, Richardson, Texas. Accessed July 19, 2013 at http://www.ices.cmu.edu/psii/nilm/slides/lai_Samsung NILM2012 slides.pdf

Lai PH, M Trayer, S Ramakrishna, and Y Li. "Database Establishment for Machine Learning in NILM, Samsung Dallas Technology Lab, Richardson, Texas. Accessed July 19, 2013 at http://www.ices.cmu.edu/psii/nilm/abstracts/lai_Samsung_NILM2012_abstract.pdf

Liang J, KK Ng Simon, G Kendall, and JWM Cheng. 2010. "Load Signature Study - Part 1: Basic Concept, Structure, and Methodology." IEEE Transactions on Power Delivery 25 (2) (2010). IEEE Power \& Energy Society, New York, New York. Accessed July 19, 2013 at http://ieeexplore.ieee.org/stamp/stamp.jsp?tp=\&arnumber $=5337912$

Marceau ML, and R Zmeureanu. 1999. "Nonintrusive load disaggregation computer program to estimate the energy consumption of major end uses in residential buildings." Department of Building, Civil and Environmental Engineering, Concordia University, Portland, Oregon. Accessed July 19, 2013 at http://spectrum.library.concordia.ca/816/ 
Marchiori A, and D Hakkarinen. 2011. "Circuit-Level Load Monitoring for Household Energy Management." Pervasive Computing, IEEE. Volume 10, Issue 1. Accessed July 19, 2013 at http://ieeexplore.ieee.org/xpls/abs_all.jsp?arnumber=5582070

Matthews HS and L Soibelman, M Berges, and E Goldman. 2008. "Automatically Disaggregating the Total Electrical Load in Residential Buildings: a Profile of the Required Solution." Proc. Intelligent Computing Engineering, 381-389. Carnegie Mellon University, Pittsburgh, Pennsylvania. Accessed July 19, 2013 at http://www.mendeley.com/research/automatically-disaggregating-total-electrical-loadresidential-buildings-profile-required-solution-1/

May S, L Fishback, D Arneman, J Cunningham, and M Jog. "Data in the Wild.” PlotWatt, Durham, North Carolina. Accessed July 19, 2013 at http://www.ices.cmu.edu/psii/nilm/abstracts/may PlotWatt NILM2012 abstract.pdf

Norford L, and S Leeb, 1996. "Non-Intrusive Electric Load Monitoring in Commercial Buildings Based on Steady-State and Transient Load-Detection Algorithms." Energy in Buildings 24 (1996) 51-64. Accessed July 19, 2013 at http://www.sciencedirect.com/science/article/pii/0378778895009582

Nunes NJ, and L Pereira. "Deploying and Evaluating the Effectiveness of Energy Eco-Feedback Through a Low-Cost NILM Solution.” M-ITI, U. Madeira Campus da Penteada, Funchal, Portugal. Accessed July 19,2013 at http://www.academia.edu/2242616/Deploying and evaluating the effectiveness of energy ecofeedback_through_a_low-cost_NILM_solution

Parson O, S Ghosh, M Weal, and A Rogers. 2012. "NIALM using Prior Models of General Appliance Types.” In Proceedings of the Twenty-Sixth AAAI Conference on Artificial Intelligence (AAAI-12). Toronto, California, July 22-26, 2012. Accessed July 19, 2013 at http://eprints.soton.ac.uk/336812/

Perez MNV. 2011. "A Non-Intrusive Appliance Load Monitoring System for Identifying Kitchen Activities." Aalto University School of Science and Technology, Finland. Accessed July 19, 2013 at http://www.diem.fi/files/news/Master Thesis Noemi Valero.pdf

Pihala H. 1998. "Non-Intrusive Appliance Load Monitoring System Based on a Modern kWh-Meter." VTT Energy, Technical Research Center of Finland, Finland. Accessed July 19, 2013 at http://www.vtt.fi/inf/pdf/publications/1998/P356.pdf

Proper E, and RW Cox. "Field Demonstration of a Real-Time Non-Intrusive Monitoring System for Condition-Based Maintenance." USCG, Massachusetts Institute of Technology, Cambridge, Massachusetts, and University of North Carolina, Chapel Hill, North Carolina. Accessed July 19, 2013 at http://esrdc.mit.edu/library/ESRDC library/MITSG 09-24.pdf

Ruzzelli AG, C Nicolas, A Schoofs, and GMP O'Hare. "Real-Time Recognition and Profiling of Appliances through a Single Electricity Sensor." Clarity: Centre for Sensor Web Technologies, School of Computer Science and Informatics, University College Dublin, Ireland. Accessed July 19, 2013 at http://ieeexplore.ieee.org/xpls/abs_all.jsp?arnumber=5508244 
Tomek S. 2012. "NIALM for Smart Buildings LoReMA Load Recognition Monitoring and Acting, iHomeLab Research Center." PowerPoint Presentation. International Workshop on Non-Intrusive Load Monitoring, May 7, 2012. Carnegie Mellon University, Pittsburgh Pennsylvania. Accessed July 19, 2013

at

http://www.ihomelab.ch/fileadmin/Dateien/PDF/NewsEvents/2012/iHomeLab_NILAM_WS_Pittsburgh_ 20120507 Tos.pdf

Tomek S, and A Andrushevich. 2012. "iHomeLab Experience with NIALM for Smart Buildings.” International Workshop on Non-Intrusive Load Monitoring, May 7, 2012. iHomeLab Research Center, Lucerne University, Luzern, Switzerland. Accessed July 19, 2013 at http://www.iotbutler.eu/publications/ihomelab-experience-with-non-intrusive-appliance-load-monitoring-nialm-forsmart-buildings

Zeifman M. 2012.“An Approximate Probabilistic Approach for Event-based Disaggregation.” International Workshop on Non-Intrusive Load Monitoring., Fraunhofer Center for Sustainable Energy Systems, Cambridge, Massachusetts. Accessed July 19, 2013 at http://www.ices.cmu.edu/psii/nilm/abstracts/zeifman_CSE_NILM2012_abstract.pdf.

Zeifman M, C Akers, and K Roth. "Nonintrusive appliance load monitoring (NIALM) for energy control in residential buildings." Fraunhofer Center for Sustainable Energy Systems, Cambridge, Massachusetts. Accessed July 19, 2013 at http://cse.fraunhofer.org/publications/eedal-2011/

Zeifman M, and K Roth. 2011. Nonintrusive Appliance Load Monitoring: Review and Outlook. IEEE Transactions on Consumer Electronics. 2011:57(1):76-84. IEEE Consumer Electronics Society, New York, New York. Accessed July 19, 2013 at http://ieeexplore.ieee.org/xpls/abs_all.jsp?arnumber=5735484

Zoha A, A Gluhak, MA Imran, and S Rajasegarar. 2012. "Non-Intrusive Load Monitoring Approaches for Disaggregated Energy Sensing: A Survey.” Sensors. 2012 Vol: 12(12) 16838:16866. Basel, Switzerland. Accessed July 19, 2013 at www.mdpi.com/1424-8220/12/12/16838.pdf 


\section{Appendix B. NILM Protocol Mechanics: Monitoring and Disaggregation Metrics}

This protocol is designed to facilitate the evaluation of a variety of the NILM technologies in a consistent, unbiased, and repeatable manner. Because of the variety of new and existing NILM offerings (both hardware and software solutions) this protocol was purposely kept general and as encompassing as possible.

The protocol was designed for technical assessment and not for assessment of market potential or acceptance. It is designed to give guidance for application and not competitive advantage or status.

\section{B.1 Definitions}

Consistent Load: Electrical loads that will engage or disengage independently of direct action from the user and are "always on." These loads may alternate between an active and inactive state as needed/required. In some instances these loads operate completely automatically and independently of the user's input, such as refrigerators or water heaters which do not require user interaction to operate as intended. Other loads operate automatically according to user-provided settings, such as resistance heaters which will activate as necessary to achieve the user's desired temperature. In both of these instances the devices are active in the background and alternating between an inactive state that uses little to no electricity, and an active state.

User-Activated Load: These loads can vary from type of appliance, but require the user to directly interact with the load in order to become active. These loads may or may not disengage themselves automatically after a set function or interval of time has passed (such as a sleep timer on a TV, or the function of a toaster), but they do not automatically enter an active state from an inactive state without user intervention.

Metering Hardware: Refers to the physical metering components that must be installed in the home to meter or record electrical load use data. This also includes any additional components, such as communications equipment or gateways that are required to transmit the collected load data to software or a vendor's remote server.

Metering Software: Any software, located either integral to the NILM or at the vendor's remote server, which processes raw electrical load use data and disaggregates these into its component electrical loads.

NILM Technology: Refers to any combination of physical hardware, metering equipment, software, or remote vendor-based disaggregation services from a single manufacturer that was sold with the explicit intention of being used to disaggregate the electrical load of a home with a single metering point.

\section{B.2 Instrumentation Specifications}

All vendor supplied metering equipment is to be installed as directed by accompanying product literature in order to test the NILM technology in a state that is as close to the manufacturer's specifications as possible. No deviation from the installation guidance is expected to ensure that the metering hardware will collect data as accurately as possible. Additionally, no calibration or alteration of either the metering 
hardware or software is expected; each technology is to be tested "as is" from the manufacturer in a state that the end-consumer would receive it. Precautions will be taken to secure the metering hardware in place where necessary, specifically for CT sensors, with plastic zip-ties to prevent sensors from shifting and interfering with any other equipment. All subsequent setup or configuration of the metering hardware will be conducted following the steps specified in the owner's documentation accompanying each technology. This also includes establishing any necessary connection between the metering hardware and associated software or vendor-based disaggregation algorithm services.

\section{B.3 Load Testing Protocol}

Load testing of individual system components will take place first to verify test equipment, communications systems, load activation procedures, and all metering. In most cases, loads will be activated automatically according to a schedule uploaded to custom electrical panel installed in each of the PNNL Lab Homes. These electrical panels, 42-breaker modified commercial lighting panels specifically designed for this application, use motorized breakers to turn on and off circuits according to a programmed schedule. Installed with this panel is a multi-channel, true-power, data logging system that affords circuit-level metering of all 42 defined breakers. This programmable panel and multi-channel data logger form the load control and the baseline reference data collection systems necessary for NILM evaluation. Appendix C provides further details on these two systems. Once all system components are verified, the individual load testing will begin first with the individual loads and then the multi-load protocols.

\section{B.3.1 Load Testing Protocol: Individual Loads}

The following steps are proposed for the individual (i.e., single load) testing procedures.

1. Verify that all loads are operating correctly prior to testing. Malfunctioning loads may not be recognizable to the meters, or individual components within the appliance or device itself may not be fully powering up, producing erratic or invalid testing results. Operate each load prior to testing and consult the home's data logging system to verify that the load in question is powering up and functioning correctly.

2. Prior to testing any appliance or electrical load, determine if the load is a consistent load or a useractivated load. Be aware that some consistent loads may require manual activation during the testing protocol, as may be the case with electric heaters if the home's temperature is above the current set point, while others will automatically engage over time. If the load is user/panel activated, note if there are a variety of modes that the user may select. Washing machines, for example, commonly have different wash modes that run shorter or longer than others, and may also have different load profiles between modes.

3. Make note of which appliances have a consistent load even when switched off by consulting the home's data logger system while testing user/panel-activated loads. These loads should be completely disengaged, either by unplugging or turning off at the breaker panel, prior to the testing of other loads as their inactive state load use may interfere with the testing of other equipment.

4. Before the testing period, note the time on the home's data logger/electrical panel display, the NILM technology's display (if present), and local accurate clock. It is important to make note of 
which time each system is displaying for reference before and after testing period to match the time stamps of the load disaggregation and sub-meter readings.

5. Activate the electrical load by turning the appliance on (or allowing the programmable panel to operate) and selecting the desired use mode (if applicable). If the appliance has multiple modes that the user is required to choose from, treat each consecutive mode individually (i.e. each mode is an individual and unique load). Do not change modes during the operation of that load unless the device or appliance does so automatically. Manually record the time that the load was powered up, as well as the time that the load ended - all testing durations will be according to a prescribed schedule by load and load type - see Section B.4.1 below for scheduling protocol.

6. After running the load for the specified duration, completely power down (or allow the panel to control) the device or appliance. If the device has an inactive state, unplug or disengage the breaker for that load to prevent its interference with other loads.

7. Execute steps 1 through 6 for all scheduled devices as part of the PNNL Lab Homes protocol, recording the times that each load was tested (beginning and end) and according to the schedule protocol in Section B.4.1.

8. Match the sub-metering data with the load disaggregation data received from the NILM vendor or vendor web site for each of the NILM technologies. These two data sets will form the basis for NILM evaluation according to evaluation metrics presented below in Section B.4.2.

\section{B.3.2 Load Testing Protocol: Multiple Loads}

The multiple load protocol will follow the same procedure as the individual load testing except specific (multiple) electrical devices will be cycled on over the testing period to verify accuracy with coincident device use.

Multiple load testing will make use of the controllable breaker panel to initiate and terminate device runtimes according to propose schedules. It is anticipated that load activation will be staggered by predetermined intervals ( 2 minutes and 10 minutes are proposed) to allow recognition in the accompanying data sets.

\section{B.4 Load Testing Protocol: Monitoring and Disaggregation Metrics}

The defined protocol will explore two key performance metrics; monitoring metrics (i.e., the ability to correctly assess load, load magnitude, and duration) and disaggregation metrics (i.e., the ability to accurately determine and classify specific loads and events). These are defined/described in the subsequent sections.

\section{B.4.1 Monitoring Metrics}

This portion of the protocol focuses on specific loads and load types, as well as load functional states. Presented below are recommended load types and proposed magnitudes. The loads are to be representative of "typical" residential equipment and have a mix of resistive and reactive power 
characteristics. The values and ranges presented are considered as "sample" magnitudes; actual magnitudes will be specific to the proposed test load and be determined at time of testing.

\section{- Low Wattage Protocol: 10 Watts to 100 Watts}

$\bigcirc$ Lighting: incandescent, fluorescent, and LED fixtures

- Home electronics: residential audio, video, set-top box devices

○ Miscellaneous plug loads: fans, kitchen appliances, etc.

\section{- Mid Wattage Protocol: 100 Watts to 1,000 Watts}

○ Lighting: multiple incandescent, fluorescent, and LED fixtures

- Home electronics: desktop computer/monitor, printer, television

○ Miscellaneous plug loads: refrigerator, clothes washer, dishwasher

\section{- High Wattage Protocol: Above 1,000 Watts}

- Lighting: multiple lighting circuits

- Home appliances: clothes dryer, water heater, HVAC

\section{B. 4.1.1 Operational Characteristics and Load Scheduling}

For each of the above wattage protocols (low, mid, and high) a schedule of load duration has been proposed. This schedule was developed based on typical residential load activation patterns, the recognition of evaluation limitations of vendor-based NILM systems, and the need for efficient testing protocols.

The evaluation load and activation schedules incorporate one-minute, ten-minute and one-hour load activations. While sub one-minute load durations were initially considered, after review of typical residential load patterns and discussions with NILM vendors it was decided that this was too narrow an interval and may be better left for a Phase 2 testing protocol.

The proposed load schedule follows a progression from the low to high wattage loads. For each of the sample loads within the three categories (low wattage, mid wattage, and high wattage), a 1-minute, 10minute and 1-hour load duration is proposed. Additionally, each load will cycle on and off at a predetermined frequency within that load duration window.

By way of example, consider the low-wattage protocol (a 25-watt incandescent lamp) and the one-minute duration schedule. This protocol is defined such that the lamp will cycle on for one minute and off for one minute. This pattern will repeat for four cycles before moving to the next low-wattage load, perhaps a 70-watt linear fluorescent fixture. After the low-wattage protocol is completed, the mid-wattage and high-wattage protocols will be executed. This progression will be complete when all loads have been cycled through the one-minute, ten-minute, and one-hour schedules.

For the multiple load protocol, the proposed schedule includes the 10-minute and 1-hour load activation intervals; the 1-minute activation with multiple loads requires too many cycles for accurate control.

\section{B.4.2 Disaggregation Metrics}


To evaluate disaggregation accuracy, a set of metrics was developed based on work proposed by Liang and Ziefman (Liang, et.al. 2010, Ziefman, et.al. 2011). The metrics proposed below allow for a comparison between NILM reported events and load magnitudes with the actual event information. The metrics are presented as a series of calculated accuracy values, specific to the event activity.

\section{A. Event Detection (single and multiple events, metric reported as percent accuracy)}

Detection Accuracy is a ratio of the correctly detected events over the total number of events detected by the NILM including the effects of wrongfully detected (false positive) events. In other words, out of all the loads the NILM detected, including false positive events, what percentage were correctly detected:

$$
\text { Detection accuracy }=\frac{\text { properly detected events }}{\{(\text { true events })+(\text { wrong events })-(\text { missed events })\}}
$$

Or-equivalently

$$
\text { Detection accuracy }=\frac{\text { properly detected events }}{\{(\text { detected events })+(\text { wrong events })\}}
$$

Where:

True events $=$ Number of actual events

Detected events $=$ Number of detected events (i.e., total detected events including false events)

Properly detected events $=$ Number of accurately detected and classified events (i.e., accurate specific event and event classification)

Wrong events $=$ Number of wrongfully detected events (i.e., false positive events)

Missed events $=$ Number of missed events (i.e., events not detected)

\section{B. Event Disaggregation (correct disaggregation of single and multiple/coincident events)}

Disaggregation Accuracy is a measure of ration of the number of events the NILM properly identified and classified over the number of events the NILM detected which were true events. In other words, out of all the loads/events the NILM detected that were real events, what percentage was disaggregated correctly? This describes how well the NILM is able to disaggregate the loads in identifies.

$$
\text { Dissagregation accuracy }=\frac{\text { properly detected events }}{\{(\text { detected events })-(\text { wrong events })\}}
$$

Or-equivalently

$$
\text { Dissagregation accuracy }=\frac{\text { properly detected events }}{\{(\text { true events })-(\text { missed events })\}}
$$




\section{Overall Event Accuracy (correct disaggregation of single and multiple/coincident events)}

Overall Accuracy is a ratio of the number of correctly identified and classified events over the number of actual events. In other words, it is a measure of "theoretical" accuracy where $100 \%$ would mean the NILM identified every real event, did not register any false positive events, and classified all events correctly.

$$
\text { Overall accuracy }=\frac{\text { properly detected events }}{\{(\text { detected events })-(\text { wrong events })+(\text { missed events })\}}
$$

Or-equivalently

$$
\text { Overall accuracy }=\frac{\text { properly detected events }}{\{\text { true events }\}}
$$

\section{Power/Energy Use. Accurate calculation of energy use (single and multiple events, metric reported as percent accuracy)}

Energy Accuracy is a measure of accurate energy use by individual appliances (single use), multiple appliances (coincident single uses), and multiple appliances over a duration (multiple uses/multiple cycles).

$$
\text { Energy accuracy }=\left[1-\left\{\frac{\text { measured energy }- \text { NILM energy }}{\text { measured energy }}\right\}\right]
$$

Where:

Measured energy $=$ energy measured by meter (watt-hours) over the defined interval

NILM energy = energy measured by NILM (watt-hours) over the defined interval

Consider the calculation of energy accuracy for:

- Single appliance and single event (fixed event duration)

- Multiple appliances and single event (fixed event duration)

- Multiple appliances and multiple events (variable event durations)

\section{E. Energy Shares. Accurate calculation of energy shares (energy use of specific event recorded as a percentage of total energy use)}

Overall Energy Share Accuracy is a measure of accurate energy use/share by individual appliances as a function of total test (i.e., multiple appliances) energy use.

$$
\text { Energy share accuracy }=\left[1-\left\{\frac{\text { measured energy share }- \text { NILM energy share }}{\text { measured energy share }}\right\}\right]
$$


Where:

Measured energy share $=\{($ measured appliance energy $) /($ measured total energy share $)$

NILM energy share $=\{($ NILM appliance energy $) /($ NILM total energy share $)$

F. Repeatability. Consistency of all evaluation criteria over multiple tests

- As time and resources permit, multiple applications of key metric evaluations will be completed to compare for consistency. 


\section{Appendix C. Controllable Electrical Panel and Reference/Baseline Metering Equipment}

\section{C.1 Controllable Electrical Panel}

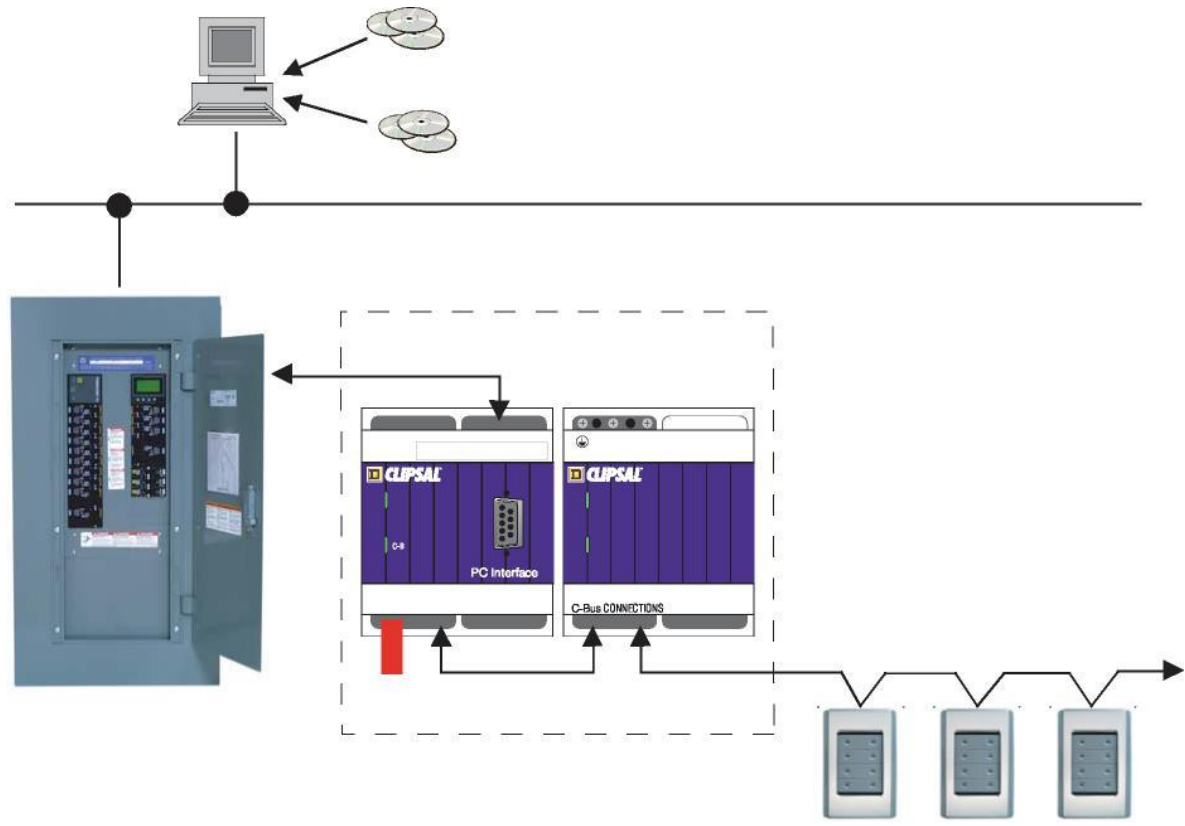

Figure C.1. Square D Power Link Electrical Panel

\section{C.1.1 Schneider Electric/Square D Power Link G3 Control System}

The Square D Powerlink G3 offers programmable and controllable breakers affording the ability to schedule on/off events at 1-minute resolution intervals.

\section{C.1.1.1 Specifications}

Square D Powerlink G3 Controllers accept commands from both dry-contact inputs and serial communications. Typical control devices include low voltage pushbutton wall switches, occupancy sensors, photo sensors, card access, and building automation systems. 
Terminals on the controller allow connection to external control devices. The 500-level controllers can accept up to eight separate control inputs, while the 1000, 2000, and 3000 level controllers can accommodate either eight 3-wire or sixteen 2-wire inputs. All controllers provide $24 \mathrm{Vdc}$ control power [National Electric Code (NEC) Class 2] for sensing input contact status. Input types include:

- maintained NO (normally open with or without blink)

- maintained NC (normally closed with or without blink)

- momentary toggle/maintained toggle

- momentary on/momentary off

- dual momentary

Controller inputs can be configured for status feedback to operate pilot lights or actuate other control equipment. A total maximum current of $60 \mathrm{~mA}$ at $24 \mathrm{Vdc}$ is available to eight status outputs.

Input timers, with settings from 1 second to 18 hours, can also be incorporated into the input configuration to provide timed override control

It combines the control, input, and scheduling features of the other systems with the added benefit of embedded Ethernet connectivity.

It provides control capabilities and communication with a true peer-to-peer (P2P) control network to allow different controllers to share input signals, schedules, and lighting zone states.

Ethernet connectivity reduces network installation costs and enhances reliability by eliminating the need for a dedicated lighting control network.

Ethernet infrastructure can also be more cost effective: it uses commonly available off-the-shelf components rather than manufacturer-specific proprietary components.

It provides enhanced system reliability: local IT staff can quickly diagnose and resolve most problems associated with the network infrastructure.

Web-enabled benefits include: ability to override zones, change schedules, and view system status from the convenience of a standard web browser.

\section{C.2 Reference/Baseline Metering Data Logger}

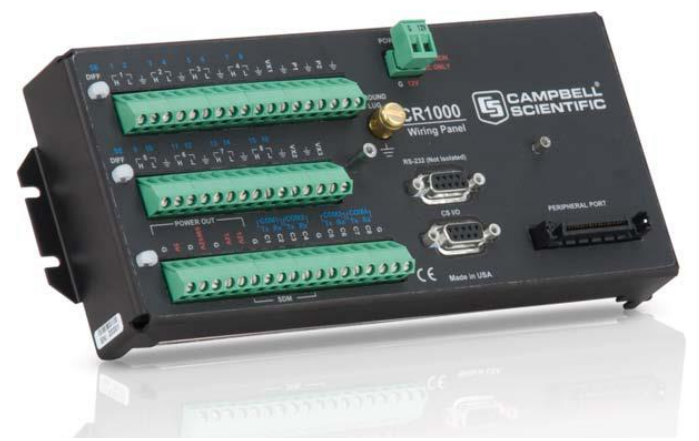

Figure C.2 Campbell CR 1000 


\section{C.2.1 Campbell CR 1000 Specifications and Features}

- 4 Mbyte memory

- Program execution rate of up to $100 \mathrm{~Hz}$

- CS input/output (I/O) and RS-232 serial ports

- 13-bit analog-to-digital conversions

- 16-bit H8S Renesas Microcontroller with 32-bit internal central processing unit (CPU) architecture

- Temperature-compensated real-time clock

- Background system calibration for accurate measurements over time and temperature changes

- Single DAC used for excitation and measurements to give ratio metric measurements

- Gas discharge tube protected inputs

- Data values stored in tables with a time stamp and record number

- Battery-backed static random access memory (SRAM) and clock maintaining data, programs, and accurate time while the CR1000 is disconnected from its main power source

- Serial communications with serial sensors and devices supported via I/O port pairs

- PakBus ${ }^{\circledR}$, Modbus, Distributed Network Protocol 3 (DNP3), Transmission Control Protocol/Internet Protocol (TCP/IP), FTP, and Simple Mail Transfer Protocol (SMTP) protocols supported

\section{C.2.2 Communications Protocols}

The CR1000 supports the PakBus, Modbus, DNP3, TCP/IP, FTP, and SMTP communication protocols. With the PakBus protocol, networks have the distributed routing intelligence to continually evaluate links. Continually evaluating links optimizes delivery times and, in the case of delivery failure, allows automatic switchover to a configured backup route.

The Modbus remote terminal unit (RTU) protocol supports both floating-point and long formats. The data logger can act as a slave and/or master.

The DNP3 protocol supports only long data formats. The data loggers are Level 2 slave compliant, with some of the operations found in a Level 3 implementation.

The TCP/IP, FTP, and SMTP protocols provide TCP/IP functionality when the CR1000 is used in conjunction with an NL115, NL120, or third-party serial internet protocol (IP) device. 



\subsection{References}

Liang J., Ng S.K.K., Kendall G., Cheng J.W.M. Load signature study Part I: Basic concept, structure, and methodology. IEEE Trans. Power Del. 2010;25:551-560.

Zeifman M., Roth K. Nonintrusive appliance load monitoring: Review and outlook. IEEE Trans. Consum. Electron. 2011;57:76-84.

Zoah, A., Alexander G, Muhammad A.I., Sutharshan R. Non-intrusive load monitoring approaches for disaggregated energy sensing: A Survey. University of Surrey, UK. 2012;1424-8220. 


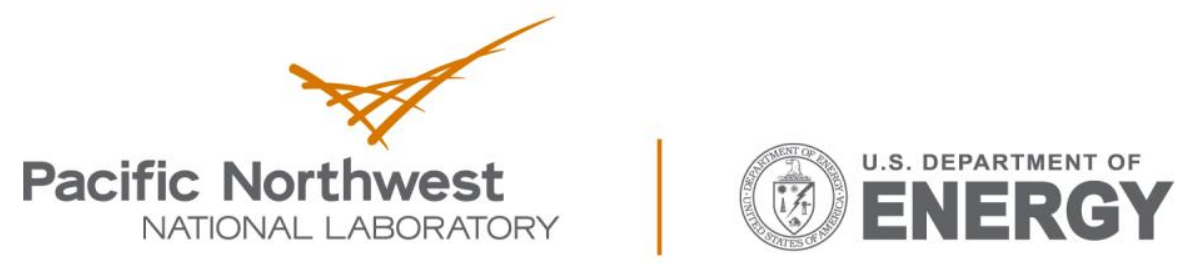

Proudly Operated by Battelle Since 1965

902 Battelle Boulevard

P.O. Box 999

Richland, WA 99352

1-888-375-PNNL (7665)

www.pnnl.gov 